\title{
Calidad educativa, apoyo docente y familiar percibido: la tridimensionalidad de la satisfacción escolar en niños y adolescentes
}

\begin{abstract}
Educational quality, perceived teacher and family support: the threedimensionality of school satisfaction in children and youth
\end{abstract}

Qualidade educacional, percepção do professor e apoio familiar: a
tridimensionalidade da satisfação escolar em crianças e adolescentes

ISSN 1688-9304 - DOI: 10.18861/cied.2020.11.2.2995

Carlos Rodríguez Garcés*1

https://orcid.org/0000-0002-9346-0780

Geraldo Padilla Fuentes**2

https://orcid.org/0000-0002-0882-1818

Marcelo Gallegos Fuentes***3

https://orcid.org/0000-0002-6153-5514

Fecha de recibido: 16/05/2019

Fecha de aprobado: 20/02/2020

\section{Resumen}

Debido a que el bienestar subjetivo de niños y adolescentes ha tomado un lugar importante para la educación este artículo analiza su nivel de satisfacción con respecto a la experiencia escolar, la calidad educativa y el apoyo percibido desde docentes y la familia. Utilizando un modelo de ecuaciones estructurales con datos de la Encuesta de Desarrollo Humano en Niños, Niñas y Adolescentes del PNUD encontramos que el sentimiento de satisfacción escolar tiene un fuerte vínculo con la participación y el sentimiento de seguridad en la escuela. Asimismo, el apoyo percibido de profesores y padres refuerza las condiciones de educabilidad y las habilidades para enfrentar desafíos académicos y sociales. La satisfacción escolar, expresión del bienestar subjetivo, sería resultado del equilibrio dinámico entre recursos escolares, pedagógicos y familiares. Lo sustancial no solo es formar parte de un lugar sino poder colaborar y construir un ambiente agradable donde convivir y sentirse emocionalmente a salvo.

Palabras clave: bienestar subjetivo, satisfacción escolar, clima de convivencia, apoyo familiar percibido, apoyo docente percibido.

\begin{abstract}
Taking into account that the subjective well-being of children and teenagers has gained an important place in the educational field, this article analyzes their level of satisfaction as regards school experience, educational quality and perceived support from teachers and family. Using a model of structural equations with data obtained from the Human Development Survey in Boys, Girls and Teenagers conducted by UNDP, we found that the feeling of school satisfaction is strongly connected with participation and feeling of safety at school. Moreover, the perceived support from teachers and parents reinforces the conditions of educability and skills to face academic and social challenges. School
\end{abstract}


satisfaction as an expression of subjective well-being would be the result of a dynamic balance among school, pedagogical and family resources. The important thing is, not only to be part of a certain place, but also to be able to collaborate and build up a pleasant environment where to live and feel emotionally safe.

Keywords: subjective well-being, school satisfaction, climate of coexistence, perceived family support, perceived teaching support.

\section{Resumo}

A Lei Geral de Educação n 18.437 (LGE), aprovada em 2008, levou a mudanças institucionais substanciais no contexto do ensino superior (ES). No campo da avaliação, surgem desenvolvimentos como a criação do Instituto Nacional de Avaliação Educacional (INEEd) ou a recente criação do Instituto Nacional de Acreditação e Avaliação do Ensino Superior (INAEET). Em face desse cenário, decorrida uma década do início deste processo, a discussão de dimensões e indicadores para a avaliação do ES a partir de uma abordagem sistêmica é mais do que pertinente. Nesse sentido, com base em uma revisão bibliográfica sobre os tópicos no campo da ES, sete dimensões são propostas para abordar a avaliação na ES de uma perspectiva sistêmica. Estes incluem: desenhos institucionais, o fator territorial que se torna mais relevante no marco do processo de descentralização experimentado pela política educacional em um nível superior, aspectos do processo sociodemográfico e educacional, questões relacionadas ao currículo, dimensão orçamentária e o crescente processo de internacionalização. Através da análise documental de relatórios e leis institucionais, entre outras, essas dimensões são descritas para o caso uruguaio. Embora o processo de diversificação institucional experimentado no campo da ES abranja o desenvolvimento de estratégias específicas pelas instituições, acreditamos que o principal desafio está em obter consenso entre as instituições do sistema para poder avançar na avaliação das dimensões consideradas.

Palavras-chave: bem-estar subjetivo, satisfação escolar, clima de convivência, suporte familiar percebido, suporte docente percebido.

\section{Introducción}

Una demanda permanente al sistema educativo chileno ha sido mejorar su calidad, pertinencia y equidad, demanda que ha sido amparada tanto por evidencias de investigación como por la efervescencia de movimientos estudiantiles que han tensionado la agenda pública. Como respuesta, los gobiernos de turno, con acento variado, han dispuesto medidas para el control de los procesos de Formación Inicial Docente afinando aspectos tales como requisitos de ingreso y evaluación, perfeccionamiento docente y aumento de rentas, mejoramiento de las condiciones de educabilidad en términos de infraestructura e insumos y reingeniería de aspectos curriculares. En lo inmediato esto ha significado un aumento de la cobertura y retención, especialmente en los sectores más vulnerables (OCDE, 2004; Redondo, 2005), aunque los logros han sido insuficientes (López, 2010), en particular si se desean potenciar mayores competencias y destrezas académicas en conjunto con el desarrollo de habilidades sociales blandas de carácter transversal. Solo en la medida en que se alcance tal propósito la institución educativa se convertirá en un espacio de aprendizaje integral que imbrique elementos éticos, afectivos, cognitivos y artísticos (Agencia de Calidad de la Educación, 2016), un lugar donde los niños y adolescentes se encuentren intelectualmente desafiados pero emocionalmente a salvo y satisfechos.

Aunque se evidencia una tradicional tendencia a poner el acento en resultados académicos medidos por pruebas estandarizadas, el concepto de calidad es polisémico y complejo (Torche, Martínez, Madrid y Araya, 2015). En la actualidad su definición operacional procura dar cuenta de un conjunto de características, indicadores y cualidades más específicos desde una perspectiva multidimensional (Brunner y Elacqua, 2004; Tedesco, 
2009; Treviño et al., 2010; Bürgi y Peralta, 2011) que captura el valor agregado generado por la intervención pedagógica y su contribución al enriquecimiento de las experiencias sociales y de aprendizaje (Bondarenko, 2007). Ello releva la importancia de los componentes intersubjetivos en el proceso de aprendizaje en el entendido de que una vez cubiertos los recursos mínimos necesarios, son los procesos psicosociales y organización interna los que condicionan la eficiencia educativa de la escuela y permiten diferenciar unos de otros en su configuración y resultados (Cornejo y Redondo, 2001).

El aprendizaje se articula y construye en un espacio de organización, seguridad y respeto donde intervienen el profesor, los alumnos, el contexto y las relaciones que logran construir (Del Rey, Ortega y Feria, 2009; Caballero, 2010), espacio de intersubjetividad de mutua afectación (Martínez, 2007) que delimita el grado de satisfacción cognitiva y emocional que los estudiantes alcanzan con su experiencia educativa y que repercute, asimismo, en los niveles de motivación, retención y logro exhibido (Osorio y Pérez, 2010; Garbanzo, 2007). Al respecto varios investigadores han cubierto la relación satisfacción-escuela en estudiantes (Huebner, Valois, Paxton y Drane, 2005; Casas et al., 2014; Alfaro et al., 2016) y han reportado una complementariedad entre ambos factores donde a mayor satisfacción mejores calificaciones y desarrollo socioemocional, crece el interés por la tarea así como la autoeficacia percibida frente a los desafíos (Mesurado, 2010) y se registra una menor prevalencia de conductas problemáticas.

La satisfacción con la escuela y el sentirse a gusto con la experiencia desarrollada en ella serían expresión de bienestar subjetivo en estudiantes, entendido este como el grado en que una persona juzga en términos positivos y de modo general su vida, tanto por la cercanía entre sus aspiraciones y logros como por el nivel de sintonía entre los sentimientos, el estado de ánimo y las oportunidades percibidas que brinda la sociedad donde se vive (PNUD, 2012).

La importancia que revisten el bienestar subjetivo y la satisfacción con respecto a la experiencia escolar posiciona al estudiante como un agente activo y capaz de evaluar el clima en que convive y los factores que lo influyen. Ello es un eje clave para la definición de prioridades institucionales (Rocha, Hernández, Mantecón y Chávez, 2017) ya que proyecta el compromiso individual por alcanzar un desarrollo personal y social óptimo. Es una evaluación cognitiva que los estudiantes realizan de su vida escolar según las oportunidades de adaptación, afiliación, participación y motivación que entrega la escuela (Casas et al., 2014) y sirve de proxy para medir la capacidad del colegio para responder a necesidades y cubrir expectativas (Surdez, Sandoval y Lamoyi, 2018).

Uno de los elementos relevantes es la percepción que se tiene del docente, tanto en sus ámbitos de competencias pedagógico-disciplinares como en su capacidad para generar relaciones de confianza, comunicación y respeto. La calidad del vínculo profesor-alumno, el clima áulico y el apoyo docente percibido condicionan favorablemente tanto los resultados de aprendizaje e integralidad del proceso educativo (Murillo, 2003; Albornoz, Silva y López, 2015) como la permanencia escolar (Rosli y Carlino, 2015). La calidad de la experiencia y satisfacción educativas, en tanto fenómenos co-construidos de interacción, puede ser nutrida o tensionada con base en las habilidades y predisposición docentes. En efecto, las creencias generales y perfiles actitudinales que tienen los profesores sobre sí mismos y sus alumnos delimitan la cobertura y profundidad curricular, condicionan el ejercicio de aula, los niveles de innovación y apropiación tecnológica y configuran sus expectativas docentes, clima áulico y valoración del esfuerzo desplegado por sus alumnos (Griffiths, Gore y Ladwig, 2006; Torche et al., 2015; Estévez et al., 2008; Aron, Milicic y Armijo, 2012; Paneiva, Bakker y Rubiales, 2018). 
En este contexto, por una parte, el apoyo docente percibido ayuda a garantizar tanto la efectividad de la intervención pedagógica como la instalación de conductas prosociales y ciudadanas en el alumnado (Cava y Musitu, 2002; López de Mesa et al., 2013; Rentería et al., 2013; Treviño, Place y Gempp, 2012; Paneiva, Bakker y Rubiales, 2018).

Por otra parte, la experiencia y satisfacción educativas no se circunscriben exclusivamente a los eventos que ocurren en el ámbito escolar por cuanto reciben la influencia de la institución familiar. La escuela por sí misma no puede cubrir todos los requerimientos para una formación óptima de ciudadanos. En esta tarea, los padres y apoderados son agentes primordiales (De León, 2011; Martínez, Romero y Vázquez, 2017). En efecto, la calidad educativa del hogar, y en particular la implicación parental, incide sobre la capacidad de la escuela para instalar valor agregado, potenciando habilidades cognitivas y prosociales (Villarroel y Sánchez, 2002; Verdugo y Rodríguez, 2009; Izar de la Fuente, Rodríguez y Escalante, 2019). Alumnos que perciben a sus padres como agentes involucrados en su proceso educativo, ya sea colaborando en tareas, participando en actividades escolares, fijando normas y horarios o implementando estrategias dialógicas en la resolución de conflictos consiguen mejores niveles de satisfacción, seguridad, motivación y rendimiento (Pascual, 2006; Binaburo y Muñoz, 2007; Del Rey, Ortega y Feria, 2009; Gázquez, Pérez y Carrión, 2011; Castro et al., 2012; Vera, González y Hernández, 2014).

En síntesis, escuela y familia son espacios interdependientes con un rol sustancial tanto en la instalación de habilidades cognitivas y prosociales como en el perfilamiento de los niveles de bienestar subjetivo de niños y adolescentes (Antonio-Aguirre, Rodríguez-Fernández y Revuelta, 2019). Por tanto, a los fines de esta investigación resulta de interés conocer, desde la perspectiva del estudiante, cómo se configura el apoyo docente y familiar percibido, cómo evalúan la calidad educativa y, por último, cómo este tridente de factores inflluye articuladamente en los niveles de satisfacción con respecto a la experiencia escolar.

\section{Metodología \\ Instrumento}

Esta investigación hace uso de los resultados de la Primera Encuesta de Desarrollo Humano en Niños, Niñas y Adolescentes (EDH-NNA). Esta encuesta aplicada en Chile en el año 2017 por el Programa de Naciones Unidas para el Desarrollo (PNUD) y el Consejo Nacional de la Infancia busca exponer la perspectiva que jóvenes de entre 12 y 17 años tienen de su experiencia vital y cómo evalúan las condiciones que la sociedad está generando para su bienestar.

Alineada con la Convención sobre los Derechos del Niño, la EDH-NNA forma parte programática de la Política Nacional de Niñez y Adolescencia definida por el Gobierno de Chile en el anhelo de transitar hacia un paradigma más integral y centrado en la protección de los derechos de la niñez y la adolescencia. En tal sentido se constituye en una experiencia inédita que visibiliza, valora y promueve la expresión de los niños y adolescentes en torno a su sentir, experiencias y anhelos al permitir relevar las temáticas y situaciones que les preocupan y las que pueden ser objeto de investigación dentro del contexto educativo, así como posibilitar el monitoreo y los ajustes para una política pública más sensible a las prioridades de este grupo etario.

En particular se utilizan las baterías de ítems referidas a las siguientes dimensiones:

a) Calidad Educativa [Cal_ed]: compuesta por 5 ítems de respuesta graduada en 4 niveles de acuerdo, los cuales miden la percepción que niños y adolescentes tienen respecto de 
las oportunidades de desarrollo integral que ofrece el establecimiento educativo al cual asisten. Indaga sobre si la escuela: entrega buena educación a sus estudiantes [ce1]; da la posibilidad de elegir actividades que sean de tu interés [ce2]; tiene los recursos necesarios para que los estudiantes aprendan [ce3]; incentiva a los estudiantes a valorar la diversidad [ce4]; incentiva la participación y organización de los estudiantes [ce5].

b) Apoyo Docente Percibido [ADP]: compuesta por 5 sentencias de respuesta graduada en 5 niveles y que explora entre niños y adolescentes la frecuencia con la que sus profesores manifiestan conductas prosociales que favorecen el aprendizaje y el clima áulico. Las mismas son: te tratan con respeto y amabilidad [ad1]; te motivan a seguir aprendiendo [ad2]; confían en tus capacidades [ad3]; permiten que expreses opiniones [ad4]; consideran tus aportes y sugerencias [ad5].

c) Apoyo Familiar Percibido [AFP]: compuesta por 9 ítems con 5 niveles de respuesta referentes a la frecuencia con que los padres expresan hacia el niño o adolescente conductas de afecto, apoyo y preocupación. En particular: reconocen las cosas buenas que haces [af1]; preguntan cómo estás y cómo te sientes [af2]; animan y apoyan para que te vaya bien en la escuela [af3]; consideran tus ideas y opiniones para tomar decisiones familiares [af4]; confían en tus capacidades para lograr tus metas escolares [af5]; hacen sentir protegido/a y seguro/a [af6]; hacen sentir querido/a [af7]; apoyan tus proyectos y cosas que quieres hacer [af8]; dicen que se sienten orgullosos de lo que haces [af9].

d) Satisfacción Escolar [Sat_Esc]: sentencia que, con una intensidad graduada en 10 niveles mide el nivel de bienestar subjetivo que los niños y adolescentes manifiestan respecto de la experiencia en su escuela. A fines analíticos esta variable adquiere un rol dependiente en el modelamiento estadístico de ecuaciones estructurales.

\section{Muestra}

Mediante un muestreo trietápico y probabilístico de estudiantes de $7 .^{\circ}$ básico a $3 .^{\circ}$ medio del territorio nacional esta encuesta comprende un total de 3.073 niños y adolescentes distribuidos en 272 establecimientos educativos (municipales, particulares subvencionados y particulares pagados). Se reporta un margen de error de \pm 1.8 puntos porcentuales con un nivel de confianza del 95\%.

Cumpliendo con criterios metodológicos de ajuste de la muestra se excluyen los casos que no tuviesen serie completa en el conjunto de ítems analizados, lo que da lugar a una muestra efectiva de 2966 casos (96.5\% de la muestra original).

En su mayor parte son estudiantes de zonas urbanas (88.8\%) de la rama científico- humanista (92.4\%). Un tercio cursa estudios secundarios (33.8\%) con una leve predominancia del sexo masculino (52.9\%). El 50.7\% asiste a colegios particulares subvencionados mientras que el $43.9 \%$ lo hace a establecimientos públicos.

\section{Procedimiento}

Una vez realizada la selección de series completas en las variables de interés se llevó a cabo un análisis descriptivo de carácter exploratorio con el fin de detectar presencia de datos anómalos, problemas de curtosis y simetría, además del tratamiento a datos perdidos.

El análisis de los datos se realizó en tres etapas a fin de corroborar las propiedades psicométricas de las dimensiones que componen el modelo. En primer lugar se efectuó el Análisis Factorial Exploratorio (AFE) para conciliar un modelo parsimonioso que, con base en los ítems, dé cuenta de una estructura lógica subyacente. Para esta operación se utilizó un estimador WLSM en razón de la cantidad de sujetos (Brown, 2006), una rotación GEOMIN que no asume ortogonalidad entre factores y es considerada estándar para variables categóricas ordinales (Méndez y Rondón, 2012) y la función ROW STANDARIZATION = 
CORRELATION para visualizar la correlación entre componentes. En este AFE los índices de ajuste fueron estimados mediante Error Medio Cuadrático de Aproximación (RMSEA), medida de bondad de ajuste robusta y confiable para muestras superiores a 1500 casos (Yuan, 2005), considerándose adecuados los valores inferiores a .08, aunque hay quienes lo sitúan por debajo del .05 (Herrero, 2010). Complementariamente se consideran los clásicos Índice de Ajuste Comparativo (CFI) e Î́ndice Tucker Lewis (TLI), exigiéndose en ambos casos cifras mayores a .95.

Los resultados fueron procesados mediante software Mplus v.07, programa estadístico especializado para análisis complejos, particularmente de variables latentes y de grandes bases de datos tanto de cortes transversales como longitudinales y que ofrece una amplia variedad de modelos, estimadores y algoritmos en una interfaz amigable con resultados estructurados de maneras comprensibles e interpretables (Muthén y Muthén, 2007; Muthén y Asparouhov, 2012; Geiser, 2013), arrojando para este efecto un RMSEA= .032, un CFI= .996 y un TLI= .995, expresión de buen ajuste.

En una segunda fase las cargas factoriales resultantes de AFE fueron sometidas a análisis de fiabilidad utilizando Alfa Ordinal dada la naturaleza de respuesta graduada en niveles que presentan los ítems (Elosua y Zumbo, 2008), arrojando altos índices de consistencia interna [Cal_ed=.839; $\mathrm{ADP}=.878 ; \mathrm{APF}=$.949].

Como último paso, con los ítems que sortearon exitosamente el Análisis Factorial y con base en los adecuados niveles de ajuste se procede a estimar mediante Ecuaciones Estructurales el modelamiento teórico propuesto. Esta es una técnica multivariante, cuya utilización en la investigación educacional se ha incrementado en el último tiempo en el interés de poner a prueba modelos teóricos que de manera más intuitiva permitan identificar los factores latentes e interacciones que definen los contextos educativos, las prácticas docentes y de aprendizaje (Khine, 2013; Kapucu y Bahçivan, 2015; Nagengast y Trautwein, 2016; Rigo y Donolo, 2018). En lo específico en este artículo se crea, en una sintaxis pertinente y parsimoniosa en Mplus v.07, un modelo de ecuaciones estructurales que busca determinar cómo las variables latentes que expresan las dimensiones Calidad educativa [Cal_ed], Apoyo Docente Percibido [ADP] y Apoyo Familiar Percibido [AFP] inciden sobre la Satisfacción Escolar [Sat_Esc] percibida.

En síntesis, mediante procedimientos de Ecuaciones Estructurales se analiza el ajuste de los datos observados con el siguiente diagrama hipotetizado: 
Diagrama 1: Modelo teórico propuesto

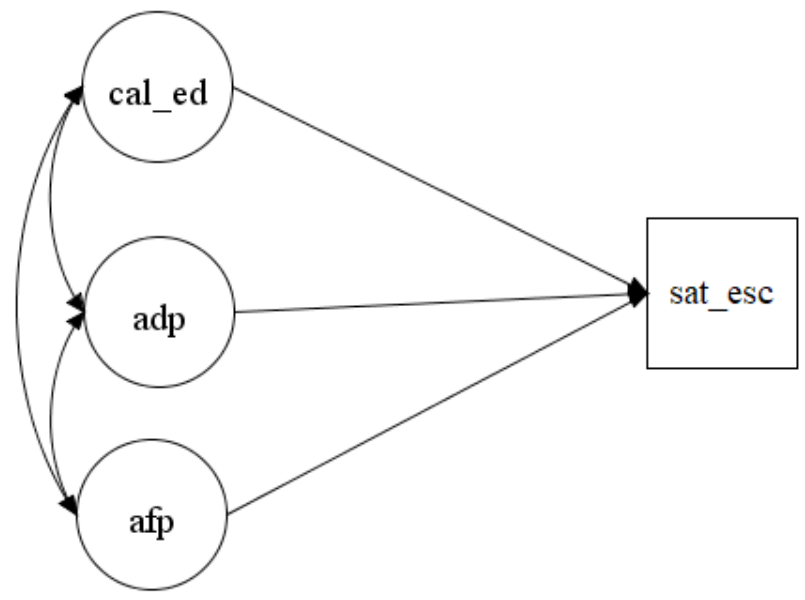

\section{Resultados}

La satisfacción escolar, variable medida en una escala de 10 niveles de graduación reporta, en términos generales, adecuadas valoraciones $(8.66+/-1,59)$, con diferencias estadísticamente significativas en favor de los estudiantes varones (8.79+/- 1.47), de Ciclo Básico (8.75 +/- 1.61) y de colegio municipal $(8.76+/$ - 1.59), aunque tales significancias influidas por tamaño de muestra resultan ser leves y escasamente relevantes al estimar el efecto controlado por tamaño de muestra. No obstante, 1 de cada 5 niños y adolescentes no está satisfecho con su experiencia en la escuela (18\%) y son especialmente críticos los provenientes de colegios particulares pagados y las mujeres.

Tabla 1. Descriptores para Satisfacción Escolar

\begin{tabular}{|l|c|c|}
\hline & Media (d.s) & \% Alta satisfacción \\
\hline Nivel Educativo & & \\
- Básico & $8.75(1.61)$ & 83.2 \\
- Medio & $8.49(1.54)$ & 79.6 \\
\hline Sexo & & \\
- Masculino & $8.79(1.47)$ & 85.0 \\
- Femenino & $8.53(1.71)$ & 78.7 \\
\hline Dependencia & & \\
- Municipal & $8.76(1.59)$ & 83.4 \\
- Part. subvencionado & $8.60(1.58)$ & 81.4 \\
- Part. pagado & $8.49(1.69)$ & 76.6 \\
\hline & $8.66(1.59)$ & $82.0 \%$ \\
\hline
\end{tabular}

Nota: Alta satisfacción reúne valores de respuesta entre 8 y 10.

Fuente: DDH-NNA (2017). Elaboración propia.

En el entendido de que el bienestar subjetivo que entrega una adecuada satisfacción escolar está influenciado por factores contextuales vinculados con la percepción de Calidad Educativa, Apoyo Docente y Apoyo Familiar, se estimaron tales influencias a través de un modelo SEM. 
Diagrama 2: Modelo explicativo para Satisfacción Escolar

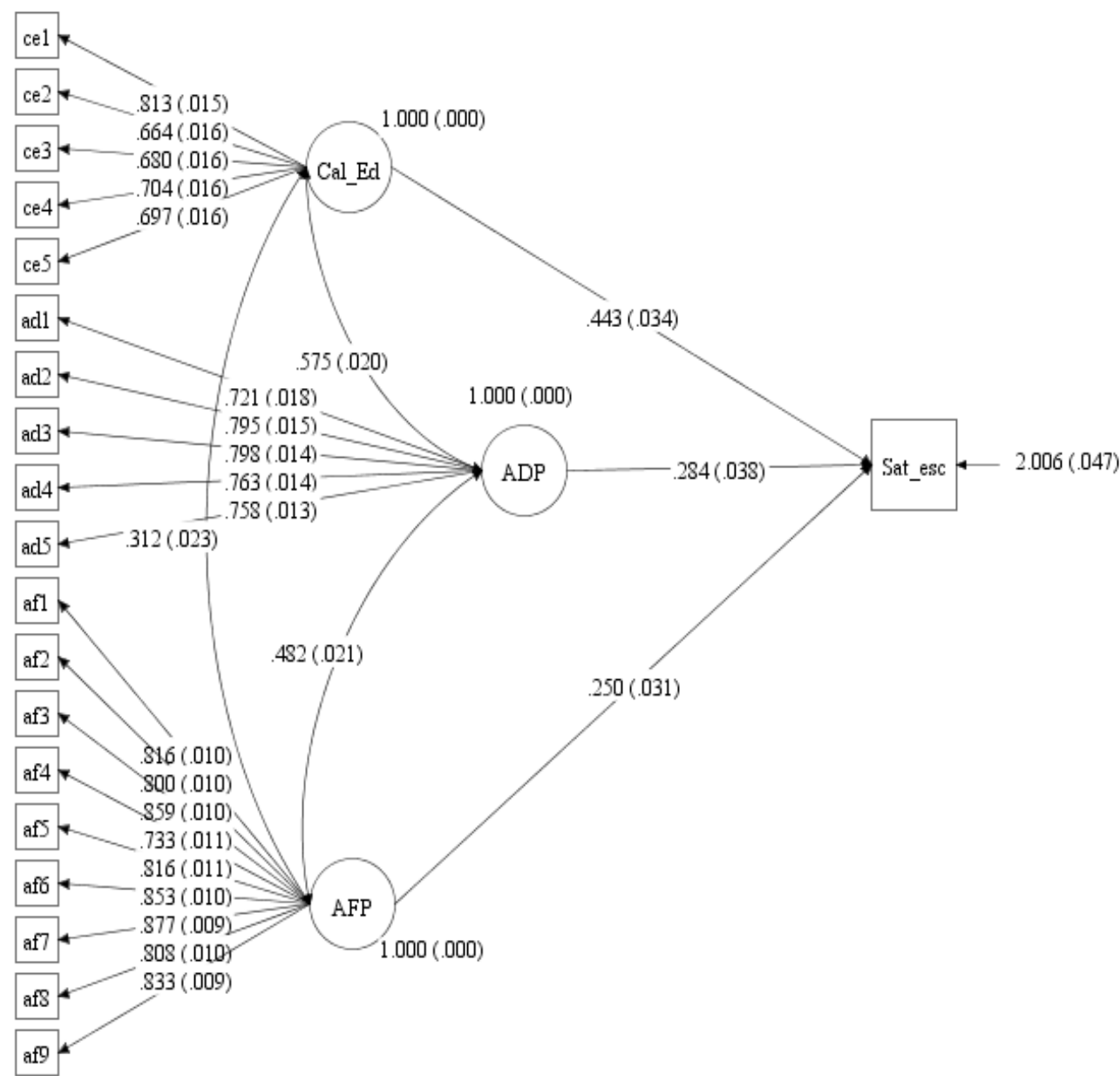

El modelo cuenta con niveles adecuados de ajuste $(\chi 2(190)=111545.530, p=.000, \chi 2 / d f$ $=587.08$, RMSEA $=.034, \mathrm{CFI}=.995, \mathrm{TLI}=.994)$.

Fuente: EDH-NNA (2017). Elaboración propia.

En el diagrama se observa que los ítems registran altas cargas con sus respectivos factores. En la dimensión Calidad Educativa (Cal_Ed) las cargas de los ítems estructurantes oscilan entre 0.664 y 0.813 mientras que los ítems vinculados al apoyo externo evidencian valores que transitan entre 0.721 y 0.798. para Apoyo Docente Percibido (ADP) y entre 0.733 a 0.877 para Apoyo Familiar Percibido (AFP).

El análisis descriptivo de los ítems incorporados al modelo evidencia altos índices de conformidad con la Calidad Educativa (Cal_Ed) de los establecimientos a los que se asiste. Al respecto los niños y adolescentes señalan que dichos centros entregan buena educación (95.7\%), otorgan la posibilidad de elegir actividades de interés (88.0\%), cuentan con los recursos adecuados para aprender (93.2\%), promueven la diversidad (93.5\%) e incentivan la participación (92.5\%).En forma análoga, el apoyo que entregan los docentes en el colegio (ADP) alcanza elevados niveles de aprobación entre los niños y adolescentes, quienes reportan que los tratan con respeto y amabilidad (95.1\%), los motivan a seguir aprendiendo (94\%), confían en sus capacidades (91.6\%), les permiten expresar su opinión (92.4\%) y consideran sus aportes y sugerencias (88.6\%). 
Por su parte el ámbito parental en tanto apoyo familiar percibido (AFP) no escapa a la tendencia favorable donde una proporción relevante de niños y adolescentes declara que sus padres regularmente reconocen las cosas buenas que hacen (87.6\%), preguntan cómo están y cómo se sienten (86.2\%), animan y apoyan su quehacer escolar (92.9\%), consideran su opinión en las decisiones familiares (77.2\%), confían en sus capacidades para lograr metas escolares (92.9\%), los hacen sentir seguros y protegidos (94.1\%), los hacen sentir queridos (92.5\%), apoyan sus proyectos (89.3\%) y manifiestan orgullo por lo que hacen (81.8\%).

La Calidad Educativa es el factor que mayor influencia ejerce sobre la Satisfacción Escolar (.443). También significativos pero levemente inferiores son los efectos directos del Apoyo Docente Percibido (.284) y del Apoyo Familiar Percibido (.250). En todos estos factores la influencia sobre la Satisfacción Escolar es de carácter positivo y con bajos índices de error estimado. El diagrama también evidencia la existencia de altas correlaciones entre factores, cuyos valores transitan entre .312 (RCal_ed-AFP) y .575 (RCal_ed-ADP).

\section{Discusión}

El bienestar subjetivo es resultado del intercambio de estímulos, comunicación y expectativas que delimitan la salud psicológica y emocional por lo que entornos de convivencia que promuevan la tolerancia, respeto, autonomía y preocupación por los otros brindan condiciones favorables para el desarrollo cognitivo-relacional y el sentirse bien (Ryff, 1995). Ello significa que en el campo educativo el nivel de bienestar alcanzado por niños y adolescentes sería resultado de la naturaleza y calidad de los vínculos establecidos no solo con los compañeros y amigos sino también con los profesores, la familia y la escuela, configuración tridimensional que estaría en la base del sentimiento de satisfacción proyectado en la evaluación de sus experiencias escolares.

La percepción de los niños y adolescentes acerca de la calidad de los establecimientos a los que asisten sería reflejo de las oportunidades de participación y expresión ofrecidas como parte del quehacer institucional. Aun cuando la apreciación que vierten sobre si reciben o no buena educación puede ser objeto de discusión debido a los diferenciales escenarios que el sistema educativo devela a través de sus mediciones SIMCE (Bellei, 2013), las opiniones tienden a la homogeneidad. Según manifiestan niños y adolescentes de ciclo básico y secundario, se hallarían en lugares que propician el aprendizaje y adquisición de competencias, el desarrollo de habilidades tanto cognitivas como sociales y se les brinda la posibilidad de incorporarse a actividades que están en sintonía con sus motivaciones personales. Es una percepción relevante dado que facilitaría el sentido de pertenencia y la participación e implicación de los distintos miembros de la comunidad educativa así como se potencia un buen clima escolar y se instalan adecuadas condiciones para el aprendizaje, coordenadas que definen la capacidad que tiene la escuela para instalar valor agregado con ocasión de la acción pedagógica (López, 2010).

Otro de los componentes de la tríada que integra el diagrama de senderos es el Apoyo Docente Percibido. Aun cuando presenta una menor intensidad en comparación al factor de Calidad Educativa, el modelo reporta que en la medida en que los alumnos perciban que sus profesores de aula entablan relaciones de cordialidad y respeto, motivan la expresión de opiniones y tienen altas expectativas sobre sus capacidades se mejora la satisfacción con respecto a la experiencia escolar. La calidad del vínculo profesor-alumno es un recurso simbólico de importancia en la optimización de las condiciones para el aprendizaje en el aula y se constituye en una demanda recurrente por parte del alumnado, tanto o más significativa que la evaluación que hacen de componentes disciplinares, instruccionales o pedagógicos (Cornejo y Redondo, 2001). Instalar vínculos significativos entre actores 
de la comunidad educativa, en particular entre profesor-alumno es una tarea compleja debido a la predominancia de relaciones jerárquicas, a los límites y normas que definen la acción pedagógica, así como por el sensible campo de la evaluación de aprendizajes y consecuencias derivados de la calificación. No obstante, lograr relaciones respetuosas y cordiales otorga satisfacción y revitaliza en los alumnos su preocupación por el aprendizaje y en los profesores el compromiso con la docencia (Albornoz, Cornejo y Assaél, 2017).

Por su parte, el Apoyo Familiar Percibido expresa una buena asociación y nivel de ajuste con el modelo SEM propuesto. Así, en la medida en que los niños y adolescentes hacen una buena evaluación del nivel de acompañamiento, preocupación y confianza recibido de sus padres eso mejora significativamente su nivel de satisfacción escolar. La familia es proveedora sustancial de soporte afectivo y emocional, sentido de pertenencia y protección, cualidades que protegen frente a las experiencias del mundo social, escuela incluida, e incrementan la valoración de sí mismos (Lastre, López y Alcazar, 2017).

La percepción de los jóvenes acerca del espacio educativo y su sentimiento de bienestar son influenciados por la orientación e intensidad de los vínculos familiares. Con base en los datos analizados los estudiantes registran altas valoraciones de AFP, especialmente en lo que refiere al apoyo en actividades escolares, proyectos personales, consideración en la toma de decisiones e interés por lo que hacen. Es una cuestión relevante por cuanto la disponibilidad de apoyo familiar no solo tiene efectos sobre el éxito escolar al promover y fortalecer el ejercicio de actividades intelectuales fuera del colegio sino que además los estilos y prácticas parentales estimulan la interpretación de expectativas y creencias sobre su desarrollo personal, escolar y social (Rivera y Milicic, 2006) pues incentiva en forma directa el esfuerzo académico, la predisposición hacia la persistencia y la calidad del clima relacional.

Varios investigadores han discutido sobre la relación del vínculo familiar con el aprovechamiento educativo y el clima escolar, exponiendo que en la medida en que se manifiestan conductas de cariño, aceptación y preocupación parental mejora la capacidad de los estudiantes para hacer frente a las contingencias del mundo escolar y a los requerimientos académicos, protegiendo de la repitencia, deserción, presencia de conductas disruptivas y deterioro de la convivencia. La disponibilidad de recursos afectivos en el hogar posibilita al estudiante desplegar un mayor nivel de responsabilidad, protagonismo e involucramiento en el quehacer educativo, lo que contribuiría también a la eficiencia y efectividad del planteamiento didáctico que el profesor desarrolla en el aula (Martínez, Romero y Vázquez, 2017; Álvarez y Martínez, 2016; Vera, González y Hernández, 2014).

En virtud de lo expuesto la satisfacción escolar de los niños y adolescentes estaría estrechamente vinculada al equilibrio dinámico entre la percepción de apoyo docente, apoyo familiar y calidad educativa de los centros de enseñanza a los que asisten. Por supuesto que no es tarea sencilla lograr una armonía en las relaciones entre el espacio educativo y el familiar por cuanto ambas instituciones tienen una estructura, alcance y relaciones diferentes. No obstante, la estabilidad que se alcanza trabajando por ello es requisito para mejorar la calidad e inclusión del quehacer educativo (Calvo, Verdugo y Amor, 2016). El apoyo que los estudiantes perciben desde sus profesores para expresar ideas, organizar actividades y participar en grupos afines debería corresponderse, dentro del hogar, con la disponibilidad de compañía, interés y preocupación por lo que el alumno hace, además de contar con ambos para recibir ayuda en caso de obstáculos académicos o conflictos interpersonales. La convivencia en climas áulicos de respeto, organizados y seguros que sustenten el bienestar físico y subjetivo de los estudiantes requiere, por 
tanto, desnaturalizar hallazgos extendidos en la investigación educativa que arguyen que a menudo los padres no tienen el tiempo para involucrarse en la escuela (Gázquez, Pérez, Lucas y Fernández, 2009), que los profesores se sienten solos y desprotegidos ante la devaluación social (Esteve, 2012) y que los estudiantes sienten que no son comprendidos (Trianes et al., 2002).

\section{Conclusiones}

Nuestra naturaleza intrínsecamente social nos empuja a desarrollar una serie de habilidades emocionales que nos permiten conocer y comprender a los otros, entablando con ellos relaciones de amistad, confianza y solidaridad. El tipo y calidad del vínculo construido condicionan el bienestar subjetivo.

En el ámbito educativo, la apreciación evaluativa que se hace de la experiencia escolar y de sus niveles de satisfacción guarda íntima relación con la disponibilidad de apoyo en otros significativos que van más allá de los compañeros y amigos. En este contexto, el apoyo familiar y docente percibido es recurso indispensable para afrontar los desafíos académicos, personales y relacionales connaturales a la acción formativa.

En este sentido, el modelo de ecuaciones estructurales analizado arrojó significativas relaciones entre los factores de percepción estudiantil en Calidad Educativa [Cal_Ed], Apoyo Docente [ADP] y Apoyo Familiar [AFP] respecto de la Satisfacción Escolar [Sat_Esc]. Conforme aumenten en frecuencia e intensidad las acciones de soporte, acompañamiento y preocupación por parte de los padres y profesores se incrementa el sentimiento de bienestar en niños y adolescentes que transitan por educación básica y secundaria. De igual modo, cuando el establecimiento se percibe como espacio organizado, seguro y de respeto, mejora la satisfacción educativa.

Estas dimensiones donde se desenvuelve el estudiante conforman pilares para el fortalecimiento del bienestar psicológico, lo que apareja recompensas en la autoestima, motivación y sentido de pertenencia al lugar en el que conviven, sea la escuela junto a compañeros y profesores, sea su hogar junto a sus padres. En la medida en que la escuela incentiva la participación y desarrolla estilos de convivencia inclusivos y, por su parte, los alumnos perciben un involucramiento parental y docente en su trayectoria, mejoran significativamente las condiciones para el aprendizaje y la capacidad para instalar competencias prosociales y de educación ciudadana, rol intrínseco de la escuela bajo un paradigma de educación integral.

\section{Referencias bibliográficas}

Agencia de Calidad de la Educación (2016). Sitio web de la Agencia de Calidad de la Educación. http://www.agenciaeducacion.cl/

Albornoz, N., Cornejo, R. y Assaél, J. (2017). Condicionantes estructurales del vínculo entre profesor y estudiante: un análisis de los discursos docentes en el contexto actual de reforma educativa en Chile. Forum Qualitative Social Research, 18(3). http://www.qualitative-research. net/index.php/fqs/article/view/2632/4142

Albornoz, N., Silva, N. y López, M. (2015). Escuchando a los niños: significados sobre aprendizaje y participación como ejes centrales de los procesos de inclusión educativa en un estudio en escuelas públicas en Chile. Estudios Pedagógicos (Valdivia), 41 (especial), 81-96. https://doi.org/10.4067/S0718-07052015000300006

Alfaro, J., Guzmán, J., Reyes, F., García, C., Varela, J. y Sirlopú, D. (2016). Satisfacción global con la vida y satisfacción escolar en estudiantes chilenos. Psykhe (Santiago), 25(2), 1-14. https://doi.org/10.7764/psykhe.25.2.842 
Álvarez Blanco, L. y Martínez-González, R. A. (2016). Cooperación entre las familias y los centros escolares como medida preventiva del fracaso y del riesgo de abandono escolar en adolescentes. Revista latinoamericana de educación inclusiva, 10(1), 175-192. https://doi. org/10.4067/S0718-73782016000100009

Antonio-Aguirre, I., Rodríguez-Fernández, A. y Revuelta, L. (2019). El impacto del apoyo social y la inteligencia emocional percibida sobre el rendimiento académico en educación secundaria. European Journal of Investigation in Health, Psychology and Education, 9(2), 109118. https://dialnet.unirioja.es/servlet/articulo?codigo $=7109638$

Aron, A. M., Milicic, N. y Armijo, I. (2012). Clima Social Escolar: una escala de evaluación -Escala de Clima Social Escolar, ECLIS-. Universitas Psychologica, 11(3), 803-813. http://www. scielo.org.co/scielo.php?script=sci_arttext\&pid=S1657-92672012000300010\&lng=en\&nrm =iso\&tlng=es

Bellei, C. (2013). El estudio de la segregación socioeconómica y académica de la educación chilena. Estudios Pedagógicos, 39(1), 325-245. https://scielo.conicyt.cl/pdf/estped/v39n1/ art19.pdf

Binaburo, J. y Muñoz, B. (2007). Educar desde el conflicto: guía para la mediación escolar. Barcelona: CEAC. http://carei.es/wp-content/uploads/educardesdeelconflicto.Gu\%C3\%ADa-para-la-mediaci\%C3\%B3n-escolar.-Andaluc\%C3\%ADa.pdf

Bondarenko Pisemskaya, N. (2007). Acerca de las definiciones de la calidad de la educación. Educere, 39, 613-621. https://www.redalyc.org/pdf/356/35603905.pdf

Brown, T. (2006). Confirmatory factor analysis for applied research. New York: Guildford. http://www.kharazmi-statistics.ir/Uploads/Public/book/Methodology\%20in\%20the\%20 Social\%20Sciences.pdf

Brunner, J. J. y Elacqua, G. (2004). Factores que inciden en una educación efectiva. Evidencia internacional. Revista virtual La Educación. http://www.educoea.org/portal/bdigital/laeducacion/139/pdfs/139pdf1.pdf

Bürgi, J. y Peralta, M. (2011). El concepto de calidad educativa en las investigaciones sobre educación en Chile (2008-2008). Revista Iberoamericana sobre calidad, eficacia y cambio en la educación [en línea], 9(3), 72-93. https://www.redalyc.org/pdf/551/55119880005.pdf

Caballero, M. (2010). Convivencia escolar. Un estudio sobre buenas prácticas. Revista de paz y conflictos (3), 154-168. https://revistaseug.ugr.es/index.php/revpaz/article/view/448

Calvo, M. I., Verdugo, M. Á. y Amor, A. M. (2016). La participación familiar es un requisito imprescindible para una escuela inclusiva. Revista Latinoamericana de Educación Inclusiva, 10(1), 99-113. https://doi.org/10.4067/S0718-73782016000100006

Casas, F. et al. (2014). Satisfacción escolar y bienestar subjetivo en la adolescencia: poniendo a prueba indicadores para su medición comparativa en Brasil, Chile y España. Suma Psicológica, 21, 70-80. https://www.sciencedirect.com/science/article/pii/ $\underline{\mathrm{S} 0121438114700098}$ 
Castro Carrasco, P. J. et al. (2012). Teorías subjetivas de profesores sobre la motivación y sus expectativas de éxito y fracaso escolar. Educar em revista (46), 159-172. https://www. scielo.br/scielo.php?pid=S0104-40602012000400012\&script=sci_arttext

Cava, M. y Musitu, G. (2002). La convivencia en la escuela. Barcelona: Paidós.

Cornejo, R. y Redondo, J. M. (2001). El clima escolar percibido por los alumnos de enseñanza media: Una investigación en algunos liceos de la Región Metropolitana. Última Década, 9(15). https://doi.org/10.4067/S0718-22362001000200002

De León Sánchez, B. (2011). La relación familia-escuela y su repercusión en la autonomía y responsabilidad de los niños/as. XII Congreso Internacional de Teoría de la Educación. Barcelona: Universitat de Barcelona. http://extension.uned.es/archivos publicos/webex actividades/4440/larelacionfamiliaescuelaysurepercusionenlaautonomiay.pdf

Del Rey, R., Ortega, R. y Feria, I. (2009). Convivencia escolar: fortaleza de la comunidad educativa y protección ante la conflictividad escolar. Revista interuniversitaria de formación del profesorado (66), 159-180. https://dialnet.unirioja.es/servlet/articulo?codigo=3098226

Elosua, P. y Zumbo, B. (2008). Coeficientes de fiabilidad para escalas de respuesta categórica ordenada. Psicothema, 20(4), 896-901. http://www.psicothema.com/pdf/3572.pdf

Esteve, J. M. (2012). El malestar docente. Temas para la educación. Revista digital para profesionales de la enseñanza (21), 1-6. https://www.feandalucia.ccoo.es/andalucia/docu/ p5sd9615.pdf

Estévez, E., Murgui, S., Musitu, G. y Moreno, D. (2008). Clima familiar, clima escolar y satisfacción con la vida en los adolescentes. Revista Mexicana de Psicología, 25(1), 119-128. https://www.uv.es/ lisis/david/mexicana.pdf

Garbanzo Vargas, G. M. (2007). Factores asociados al rendimiento académico en estudiantes universitarios, una reflexión desde la calidad de la educación superior pública. Revista Educación, 31(1), 43-63. https://www.redalyc.org/pdf/440/44031103.pdf

Gázquez, J., Pérez, M. C. y Carrión, J. (2011). Clima escolar y resolución de conflictos según el alumnado: un estudio europeo. Revista de Psicodidáctica, 16(1), 39-58. https://www.ehu. eus/ojs/index.php/psicodidactica/article/view/1144/0

Gázquez, J., Pérez, M. C., Lucas, F. y Fernández, M. (2009). Análisis de la convivencia escolar por los docentes europeos. Aula abierta, 37(2), 11-18. https://dialnet.unirioja.es/servlet/ articulo?codigo $=3088550$

Geiser, C. (2013). Data Analysis With MPlus. New York: Guilford. ISBN 978-1-4625-0245-5.

Griffiths, T., Gore, J. y Ladwig, J. (2006). Teachers' fundamental beliefs, commitment to reform and the quality of pedagogy. Australian Association for Research in Education Annual Conference. https://www.researchgate.net/publication/260753161_Teachers' fundamental beliefs_commitment to reform_and the quality_of_pedagogy 
Herrero, J. (2010). El Análisis Factorial Confirmatorio en el estudio de la Estructura y Estabilidad de los instrumentos de evaluación: un ejemplo con el Cuestionario de Autoestima CA-14. Psychosocial Intervention, 19(3), 289-300. https://www.redalyc.org/ pdf/1798/179817507009.pdf

Huebner, E., Valois, R.F., Paxton, R.J. et al. (2005). Middle school students' perceptions of quality of life. Journal of Happiness Studies (6), 15-24. https://link.springer.com/ article/10.1007/s10902-004-1170-x

Izar de la Fuente, I., Rodríguez, A. y Escalante, N. (2019). Apoyo social percibido e implicación escolar: correlaciones y variabilidad. European Journal of Child Development, Education and Psychopathology, 7(1), 23-35. https://www.researchgate.net/publication/333450959_Apoyo_ social_percibido_e_implicacion_escolar_Correlaciones_y_variabilidad

Kapucu, S. y Bahçivan, E. (2015). High school students' scientific epistemological beliefs, self-efficacy in learning physics and attitudes toward physics: A structural equation model. Research in Science and Technological Education, 33(2), 252-267

Khine, M. (2013). Application of structural equation modeling in educational research and practice. The Netherland: Rotterdam. https://www.researchgate.net/publication/257645071 Application_of_Structural_Equation_Modeling_in_Educational_Research_and_Practice

Lastre Meza, K., López Salazar, L. D. y Alcazar Berrio, C. (2017). Relación entre apoyo familiar y el rendimiento académico en estudiantes colombianos de educación primaria. Psicogente, 21(39). https://doi.org/10.17081/psico.21.39.2825

López, P. (2010). Variables asociadas a la gestión escolar como factores de calidad educativa. Estudios Pedagógicos (Valdivia), 36(1). https://doi.org/10.4067/S0718-07052010000100008

López de Mesa, C., Soto, M. F., Carvajal, C. y Urrea, P. (2013). Factores asociados a la convivencia escolar en adolescentes. Educación y Educadores, 16(3), 383-410. https://www. redalyc.org/pdf/834/83429830001.pdf

Martínez, D. (2007). Nuevas regulaciones, nuevos sujetos. En Feldfeber, M. y Oliveira, D. A. (2009). Políticas educativas y trabajo docente: nuevas regulaciones, ¿nuevos sujetos? Buenos Aires: NOVEDUC. https://books.google.cl/books?id=k6NWd5001-QC\&pg=PA33\&lpg=PA3 $3 \& \mathrm{dq}=$ Mart\%C3\%ADnez, + D.+(2007).+Nuevas + regulaciones,+ nuevos + sujetos \&source $=\mathrm{bl}$ \&ots=uyajoPrmJx\&sig=ACfU3U3x1 byXjxxrIVG699qi5-GAyu1BHA\&hl=es\&sa=X\&ved=2ahU KEwiGsoKrgMvqAhVXHrkGHUpiD7sQ6AEwAHoECAYQAQ\#v=onepage\&q=Mart\%C3\%AD nez\%2C\%20D.\%20(2007).\%20Nuevas\%20regulaciones\%2C\%20nuevos\%20sujetos\&f=false

Martínez, José Luis Villalobos, Romero, Gabriel Antonio Flórez, \& Vásquez, David Alberto Londoño. (2017). La escuela y la familia en relación con el alcance del logro académico. La experiencia de la Institución Educativa Antonio José de Sucre de Itagüí (Antioquia) 2015. Aletheia. Revista de Desarrollo Humano, Educativo y Social Contemporáneo, 9(1), 58-75. http://www.scielo.org.co/scielo.php?script=sci_arttext\&pid=S2145-

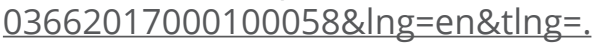


Méndez, C. y Rondón, M. (2012). Introducción al análisis factorial exploratorio. Revista Colombiana de Psiquiatría, 41(1), 197-207. https://www.redalyc.org/pdf/806/80624093014. pdf

Mesurado, B. (2010). La experiencia de Flow o Experiencia Óptima en el ámbito educativo. Revista Latinoamericana de Psicología, 42(2), 183-192. http://www.scielo.org.co/pdf/rlps/ v42n2/v42n2a02.pdf

Murillo, F. J. (Ed.). (2003). La investigación sobre eficacia escolar en Iberoamérica. Revisión Internacional sobre el estado del arte. Bogotá. https://sede.educacion.gob.es/publiventa/ descarga.action?f_codigo_agc=14324_19

Muthén, B. y Asparouhov, T. (2012). Bayesian structural equation modeling: a more flexible representation of substantive theory. Psychological Methods, 17(3), 313-335. https://doi. org/10.1037/a0026802

Muthén, L.K. and Muthén, B.O. (1998-2012). Mplus User's Guide. Seventh Edition. Los Angeles, CA: Muthén \& Muthén. https://www.statmodel.com/download/usersguide/ Mplus\%20user\%20guide\%20Ver 7_r3_web.pdf

Nagengast, B. y Trautwein, U. (2016). The prospects and limitations of latent variable models in Educational Psychology. En L. Corno y E. Anderman, Handbook of Educational Psychology (41-58). New York: Routledge. https://www.researchgate.net/publication/281030918 The prospects_and_limitations_of_latent_variable_models_in_educational_psychology

OCDE (2004): Revisión de políticas nacionales en educación: Chile. París y Santiago: OCDE y Mineduc. http://www7.uc.cl/webpuc/piloto/pdf/informe_OECD.pdf

Osorio, J. V. y Pérez, K. M. (2010). El nivel de satisfacción escolary su relación con la orientación vocacional en alumnos de psicología educativa (Tesis profesional, Universidad Pedagógica Nacional, México). http://200.23.113.51/pdf/27385.pdf

Paneiva, J. P., Bakker, L. y Rubiales, J. (2018). Clima áulico. Características socio-emocionales del contexto de enseñanza y aprendizaje. Educación y Ciencia, 7(49), 55-64. http://www. educacionyciencia.org/index.php/educacionyciencia/article/view/445

Pascual, P. (2006). Calidad, equidad e indicadores en el sistema educativo español. Pulso (29), 43-58. https://dialnet.unirioja.es/servlet/articulo?codigo=2200891

PNUD (2012). Informe de Desarrollo Humano en Chile. Bienestar subjetivo: el desafío de repensar el desarrollo. Santiago de Chile: Programa de las Naciones Unidas para el Desarrollo. https:// www.cl.undp.org/content/chile/es/home/library/human_development/publication_3.html

Redondo, J. M. (2005). El experimento chileno en educación: ¿conduce a mayor equidad y calidad en la educación? Última Década, 13(22). https://doi.org/10.4067/S0718$\underline{22362005000100005}$

Rentería, M., González, E., García, M. T. y Macías, M. (2013). El clima áulico y los factores que le afectan. Revista Iberoamericana para la Investigación y el Desarrollo Educativo (10). http://1-11.ride.org.mx/index.php/RIDESECUNDARIO/article/view/262/257 
Rigo, D. y Donolo, D. (2018). Modelos de ecuaciones estructurales. Usos en investigación psicológica y educativa. Revista Interamericana de Psicología, 52(3), 345-357. https://journal. sipsych.org/index.php/lJP/article/view/388/942

Rivera, M. y Milicic, N. (2006). Alianza familia-escuela: percepciones, creencias, expectativas y aspiraciones de padres y profesores de enseñanza general básica. Psykhe [online], 15(1), 119-135. https://scielo.conicyt.cl/scielo.php?script=sci_arttext\&pid=S071822282006000100010\&lng=es\&nrm=iso>. ISSN 0718-2228. http://dx.doi.org/10.4067/ S0718-22282006000100010.

Rocha Vázquez, M., Hernández López, M., Mantecón Ledo, M. y Chávez Amaro, D. (2017). Satisfacción de egresados cubanos de la carrera de Medicina con la formación recibida. Curso 2015-2016, Cienfuegos. MediSur, 15(4), 509-515. https://www.redalyc.org/ pdf/1800/180052835008.pdf

Rosli, N. y Carlino, P. (2015). Acciones institucionales y vinculares que favorecen la permanencia escolar de alumnos de sectores socioeconómicos desfavorecidos. Estudios Pedagógicos (Valdivia), 41(1), 257-274. https://dx.doi.org/10.4067/S0718-07052015000100015 https://scielo.conicyt.cl/scielo.php?script=sci_arttext\&pid=S0718-07052015000100015\&ln g=es\&nrm=iso\&tlng=es

Ryff, C. (1995). Psychological well-being in adult life. En Current directions in psychological science, 4(4), 99-104. https://doi.org/10.1111/1467-8721.ep10772395

Surdez, E. G., Sandoval, M. C. y Lamoyi, C. L. (2018). Satisfacción estudiantil en la valoración de la calidad educativa universitaria. Educación y Educadores, 21(1), 9-26. https://doi. org/10.5294/edu.2018.21.1.1

Tedesco, J. C. (2009). Calidad de la educación y políticas educativas. Cadernos de pesquisa, 39(138), 795-811.

Torche, P., Martínez, J., Madrid, J. y Araya, J. (2015). ¿Qué es "educación de calidad" para directores y docentes? Calidad en la educación (43), 103-135. https://doi.org/10.4067/S071845652015000200004

Treviño, E., Place, K. y Gempp, R. (2012). Análisis del clima escolar ¿poderoso factor que explica el aprendizaje en América Latina y el Caribe? Santiago: Oficina Regional de Educación para América Latina y el Caribe. OREALC/ UNESCO. http://www.unesco.org/new/fileadmin/ MULTIMEDIA/FIELD/Santiago/pdf/analisis-del-clima-escolar.pdf

Treviño, E., Valdés, H., Castro, M., Costilla, R., Pardo, C. y Donoso, F. (2010). Factores asociados al logro cognitivo de los estudiantes de América Latina y el Caribe. Santiago de Chile: OREALC/ UNESCO. https://www.researchgate.net/publication/44841035_Factores_asociados_al_ logro_cognitivo_de_los_estudiantes_de_America_Latina_y_el_Caribe

Trianes, M. V., Blanca, M. J., Muñoz, A., García, B., Cardelle-Elawar, M. \& Infante, L. (2002). Relaciones entre evaluadores de la competencia social en preadolescentes: profesores, iguales y autoinformes. Anales de Psicología, 18(2), 197-214. https://www.redalyc.org/ pdf/167/16718201.pdf 
Vera N., J., González J., C. y Hernández G., S. (2014). Familia y logro escolar en matemáticas del primer ciclo escolar de educación primaria en Sonora, México. Estudios Pedagógicos (Valdivia), 40(1), 281-292. https://doi.org/10.4067/S0718-07052014000100017 https://scielo. conicyt.cl/pdf/estped/v40n1/art17.pdf

Verdugo, M. A. y Rodríguez, A. (2009). La inclusión educativa en España desde la perspectiva de alumnos con discapacidad intelectual, de familias y de profesionales. Revista de Educación, 358, 450-470. https://knowledgesociety.usal.es/sites/default/files/La_inclusion educativa_en_Espana_desde_la_perpectiva_de_los_alumnos_con_discapacidad.pdf

Villarroel Rosende, G. y Sánchez Segura, X. (2002). Relación familia y escuela: un estudio comparativo en la ruralidad. Estudios Pedagógicos (Valdivia), (28). https://doi.org/10.4067/ S0718-07052002000100007 https://scielo.conicyt.cl/scielo.php?script=sci_arttext\&pid =S0718-07052002000100007

Yuan, K.-H. (2005). Fit indices versus test statistics. Multivariate Behavioral Research, 40(1), 115-48. DOI: 10.1207/s15327906mbr4001_5

\section{(cc) BY}

Este artículo está sujeto a una licencia internacional Creative Commons Attribution 4.0.

$\star, * *, * * *$ Universidad del Bío-Bío (Chile)

${ }^{1}$ Doctor en Multimedio Educativo, Universidad de Barcelona. Investigador, Centro de Investigación CIDCIE, Universidad del Bío-Bío (Chile).

${ }^{2}$ Licenciado en Trabajo Social, Universidad del Bío-Bío (Chile). Trabajador Social, Centro de Investigación CIDCIE, Universidad del Bío-Bío (Chile).

${ }^{3}$ Fuentes Académico departamento de Ciencias Sociales y Trabajador Social, Universidad del Bío-Bío (Chile). Investigador en sociedad, educación.

\section{Notas}

Aprobación final del artículo, editora responsable Mag. Verónica Zorrilla de San Martín.

Contribución de autoría: los tres autores han participado conjuntamente en la concepción del trabajo científico, redacción, revisión y corrección del manuscrito así como en su ajuste a estándares de calidad para la investigación social. El trabajo de construcción del modelo de datos y análisis estadístico fue especialmente orientado y realizado por Carlos Rodríguez Garcés y Geraldo Padilla Fuentes, al tiempo que Marcelo Gallegos Fuentes tuvo un papel relevante en la definición y elaboración de relaciones conceptuales y teóricas. Todos los autores han revisado y aprueban el contenido final. 